\title{
MULTIELECTRODE MICROPROBES FOR DEEP-BRAIN STIMULATION FABRICATED USING A NOVEL 3-D SHAPING ELECTROPLATING PROCESS
}

\author{
Paulo S. Motta and Jack W. Judy \\ Electrical Engineering Department, University of California, Los Angeles \\ Los Angeles, CA 90095, USA
}

\begin{abstract}
This paper describes a novel 22-mm-long multi-channel microelectrode suitable for deep-brain stimulation in rodents. The platinum electrodes and conducting interconnects are insulated by silicon nitride and the mechanical support is provided by a 3-D electroplated biocompatible metallic structure. The shape of the probe has been designed to provide sufficient mechanical stiffness for accurate targeting of deep-brain regions while minimizing tissue damage. The probe thickness and width are smoothly tapered from the $200-\mu \mathrm{m}$-wide and $150-\mu \mathrm{m}$-thick shank to the $10-\mu \mathrm{m}$-thick probe tip. The electrical and mechanical properties of the electrode array have been tested and show an impedance of $4.5 \mathrm{k} \Omega$ at $10 \mathrm{kHz}$.
\end{abstract}

\section{INTRODUCTION}

For nearly half a century [1], microprobes have been used in many different applications to study neuronal tissues and even to restore neuronal function[2]. Although much of this work has been performed in the cortex (i.e., the outer 1-mm-thick layer of the brain that is responsible for processing sensory and motor information as well as learning and memory), an exciting field of research is the recording and stimulation of deep-brain regions (i.e., the thalamus located more than $2 \mathrm{~cm}$ below the cortex). The thalamus acts as the emotional center of the brain and provides fine adjustments to the motor system. In fact, it has been shown that deep-brain stimulation (DBS) of the subthalamic nucleus can eliminate the tremors associated with Parkinson's disease [3-4].

An ideal DBS stimulation system has the following characteristics: all materials exposed to the surrounding environment are biocompatible; the stimulating parameters are such that irreversible reactions (e.g., hydrolysis or dissolution) do not occur at the electrode sites; the probe geometry provides a stiff structure to penetrate biological tissues; the tip should be narrow enough to cause the least amount of damage.

The microprobes of stimulators are typically either insulated wires or microfabricated electrode arrays. Insulated metal microwires have long been used in electrophysiology to record and stimulate neural tissue. Microwires are inexpensive, have a wide shank that is easy to handle, possess a sharp tip to minimize tissue damage, and is smoothly tapered in width and thickness to provide the stiffness needed to penetrate neural tissue deeply without bending. The major drawback of microwires is that only one electrode is available per wire. Previously reported multielectrode microprobes have been produced using the following processes and materials to form the probe shank: (1) deeply diffused $\mathrm{p}+$ wells in single crystal silicon [5], (2) the upper single-crystal silicon layer of silicon-on-insulator (SOI) wafers [6], and (3) uniformly electroplated metal [7]. In all of these probe fabrication processes, the resulting shank either has a uniform thickness - except right at the tip - or has an abrupt two-stage thickness with a thin region near to the tip and a thicker region away from the tip to facilitate easy handling. We report here a microprobe that combines the advantages of traditional microwires (i.e., tapered width and thickness) with those of microfabrication (i.e., multiple electrodes per shank) that enable a long probe to be stiff yet minimizes tissue damage upon insertion.

Multielectrode microprobes uniquely suited for deep-brain stimulation in rats have been produced using a simple low-cost batch-fabrication process. The fabricated probes have a length greater than $20 \mathrm{~mm}$ and have both a thickness and a width that can be varied smoothly from the dimensions of the tip $(\sim 10 \mu \mathrm{m})$ to that of the shank $(\sim 150 \mu \mathrm{m})$.

The complete fabrication process consists of metal traces insulated by LPCVD (bottom) and PECVD (top) silicon nitride, which forms the thin-film electrode array and interconnects. A metal seed layer is then patterned so that a 3-D structure that supports the electrode array can be formed by electroplating Figure 1.

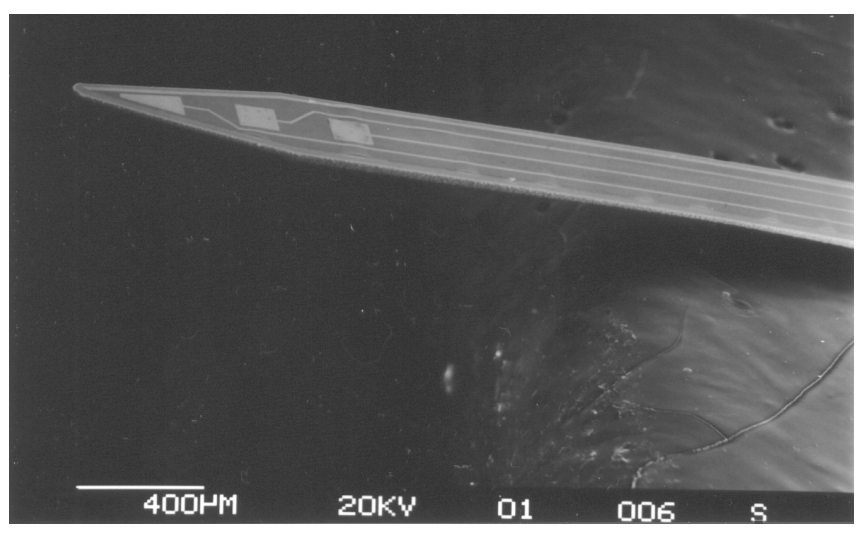

Figure 1. Released probe with 3 electrode sites.

\section{PROBE DESIGN}

The intended application largely dictates many of the probe design parameters: length, width, thickness, stiffness, and number of electrodes. For example, the length of cortical electrodes only needs to be $\sim 1 \mathrm{~mm}$ [5], whereas deep-brain stimulation-probes must be approximately 10 times longer (e.g., $\sim 10 \mathrm{~mm}$ in rats to reach the STN) plus several millimeters to allow the probe to interface with the stimulation circuitry and packaging.

Since the DBS system design for rats requires an overall probe length of $22 \mathrm{~mm}$, the mechanical stiffness of microprobes is important for accurately targeting small deep-brain regions (e.g., the rodent STN is $\left.1.0 \times 0.5 \times 0.5 \mathrm{~mm}^{3}\right)$. Cross sections of microfabricated probes are usually rectangular, semicircular, or a combination of both. To illustrate the impact of a ten-fold increase in the beam cross-section geometry, the theoretical stiffness of semicircular cross section is calculated and plotted as a function of beam length (Figure 2). For simplicity, beams with a constant cross section are considered in Figure 2. For semicircular cross sections, the beam stiffness $k_{S C}$ is given by [8] 


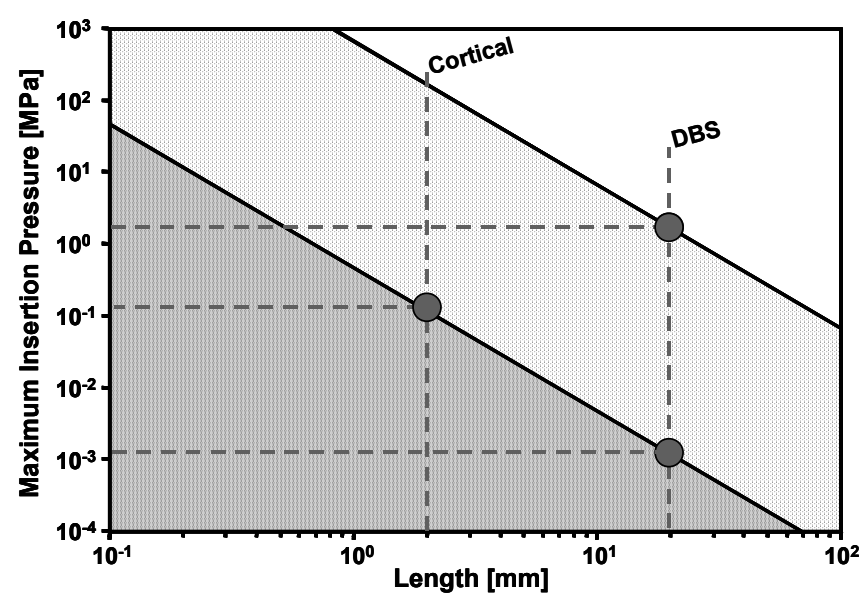

Figure 2. Plot of maximum theoretical insertion pressure as a function of probe length for those with a typical cortical and DBS probe cross sections.

$$
k_{S C}=\frac{E \cdot r^{4}}{3 \cdot l^{3}},
$$

with elastic modulus $E$, semicircular radius $r$, and length $l$. The buckling strain $\varepsilon$ of a beam fixed on one end is given by

$$
\varepsilon=\frac{\pi^{2} \cdot I}{A \cdot l^{2}}=\frac{P}{E},
$$

with semicircular cross sectional area $A$, moment of inertia $I$ and insertion pressure $P$. The maximum insertion pressure before probe buckling takes place can be calculated for each probe geometry

$$
P=\frac{\pi^{2} \cdot E \cdot I}{A \cdot l^{2}} .
$$

We see that the stiffness has a cubic dependence on length and will drop off rapidly as $l$ is increased. Since DBS probes are $\sim 10$ times longer than cortical probes, they are 1000 times less stiff for a given cross section. However, increasing the width and thickness by a factor of 10 will increase the stiffness by a factor of $10^{4}$ for a given probe length. The net result is that even though DBS probes are 10 times longer than cortical probes, they can be 10 times stiffer. Furthermore, if the cortical probes were made as long as a DBS probes, while keeping the cross sectional area unchanged, they would be able to withstand only 3 orders of magnitude less insertion pressure than DBS probes.

Besides being stiff enough to withstand insertion forces and tissue inhomogeneity, the microelectrode must cause the least amount of tissue damage at the active end (i.e., the tip). Thus the thicker and wider DBS probes must smoothly taper to a sharp tip. Other desired probe characteristics are biocompatibility and increased electrode count. Chronic probes must be made of biocompatible materials to avoid rejection of the probe by the host tissue. The greater the number of electrodes available, the larger the number of stimulation patterns possible and the greater the capability of the stimulation system.

Although microwires have been shown to be biocompatible and have a tapered geometry that enables a stiff probe that causes minimum damage, only one electrode is available per microwire. Microfabricated probes provide a high electrode count but the processes used to produce them do not allow for the tapered geometry ideally suited for DBS applications. A microprobe technology that combines the best of microwires and microfabricated probes is needed to address DBS applications, such a Parkinson's disease, epilepsy, etc. (a)

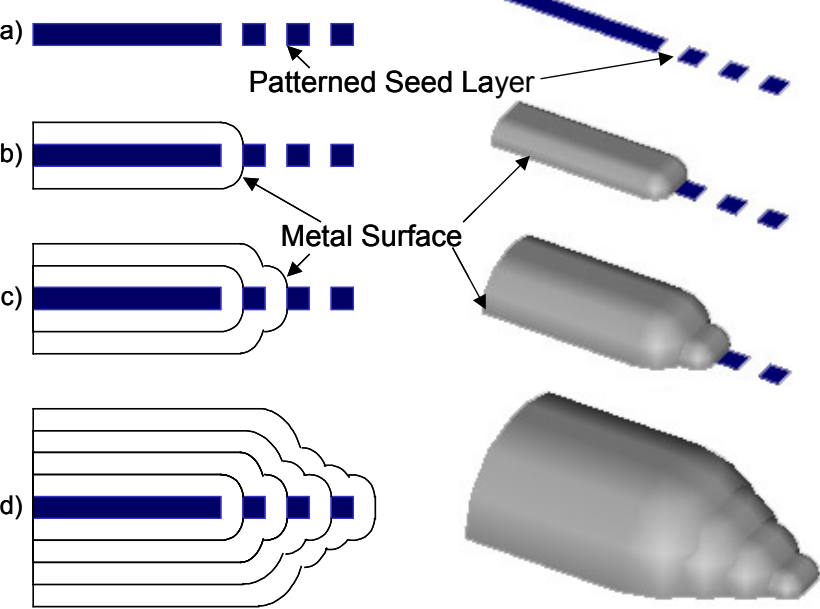

Figure 3. Schematic diagram illustrating (a) patterned seed layer before plating, (b) after the isotropically grown electrodeposit shorts to the first, (c) second, (d) all adjacent patterned seed layer regions.

\section{3-D SHAPING ELECTROPLATING PROCESS}

The microfabrication technique reported here uses electroplating to create customizable 3-D structures. Electroplating requires the presence of a seed layer, upon which the electrodeposit is formed. Usually the seed layer is continuous and a plating mold is used to determine the final shape of the electrodeposited film (i.e., through-mask plating). This method leads to sidewalls that mimic that of the plating mold and often results in vertical sidewalls and sharp corners, that are undesirable in chronic implants.

By using a patterned seed layer (i.e., temporarily electrically isolated regions of the seed layer), sidewalls with a slope of 1 or less can be achieved [9]. The electrodeposit grown on a patterned seed layer rounds sharp edges. As the electrodeposit grows vertically and horizontally from one region of patterned seed layer, adjacent regions of patterned seed layer become electrically connected due to the lateral growth. Subsequent deposition will then commence on these newly connected regions as well as the previously plated structures. As illustrated in Figure 3, this process allows for the fabrication of tapered probes which will reduce the amount of tissue damage at the stimulation site while retaining the targeting accuracy needed for long probes.

\section{FABRICATION}

The DBS mutlielectrode microprobes reported here consists of four parts: insulating layer, interconnects, electrode sites, and mechanical supporting layer. Silicon nitride deposited by LPCVD and PECVD serves as the insulating layer between the interconnects and supporting layer as well as a chemical barrier between the interconnects and surrounding tissue and fluids. Electrode sites and interconnects are made of platinum, which was chosen for its biocompatibility and charge delivery capability. The mechanical layer made of electroplated nickel and gold provides the probe with the desired level of stiffness. Starting with a $<100>$ oriented 100-mm-diameter p-type silicon wafer, $100 \mathrm{~nm}$ of lowstress LPCVD silicon nitride is deposited to serve as the first insulating layer. This layer is then patterned to define the electrode openings $\left(100 \times 100 \mu^{2}\right)$ and the probe shank width. To avoid 
spurious metal deposition during the electroplating step, a 100-nmthick layer of thermal silicon dioxide is grown. Since the previously patterned nitride serves as an oxidation barrier, the silicon dioxide only grows in the openings where silicon was exposed (Figure 5a).

In order to be able to release the microprobes from the silicon substrate, openings on the backside of the wafer must be created for the bulk release etch. In the next step, $10 \mathrm{~nm}$ of $\mathrm{Cr}$, $150 \mathrm{~nm}$ of $\mathrm{Pt}$, and another $10 \mathrm{~nm}$ of $\mathrm{Cr}$ are deposited via electron-beam evaporation, to form the probe interconnect and electrode sites (Figure 5b). Using wafer curvature techniques, the stress of the evaporated metal layer is measured to be $933.5 \mathrm{MPa}$ tensile and its sheet resistance is $1.112 \Omega /$ sq. The chromium layers serve as adhesion layers between the insulating layers and the platinum interconnect.

The top insulation layer, a 500-nm-thick silicon nitride layer, is then deposited by PECVD. The purpose of this layer is to electrically isolate the electrodes and interconnects from the mechanical supporting layer. Once the top insulator is patterned, a platinum line that surrounds the probe becomes exposed. This feature will be used during the plating of the mechanical supporting layer to completely seal in the non-biocompatible parts of the probe (i.e., $\mathrm{Cr}, \mathrm{Ni}$ ), as shown in Figure 5c.

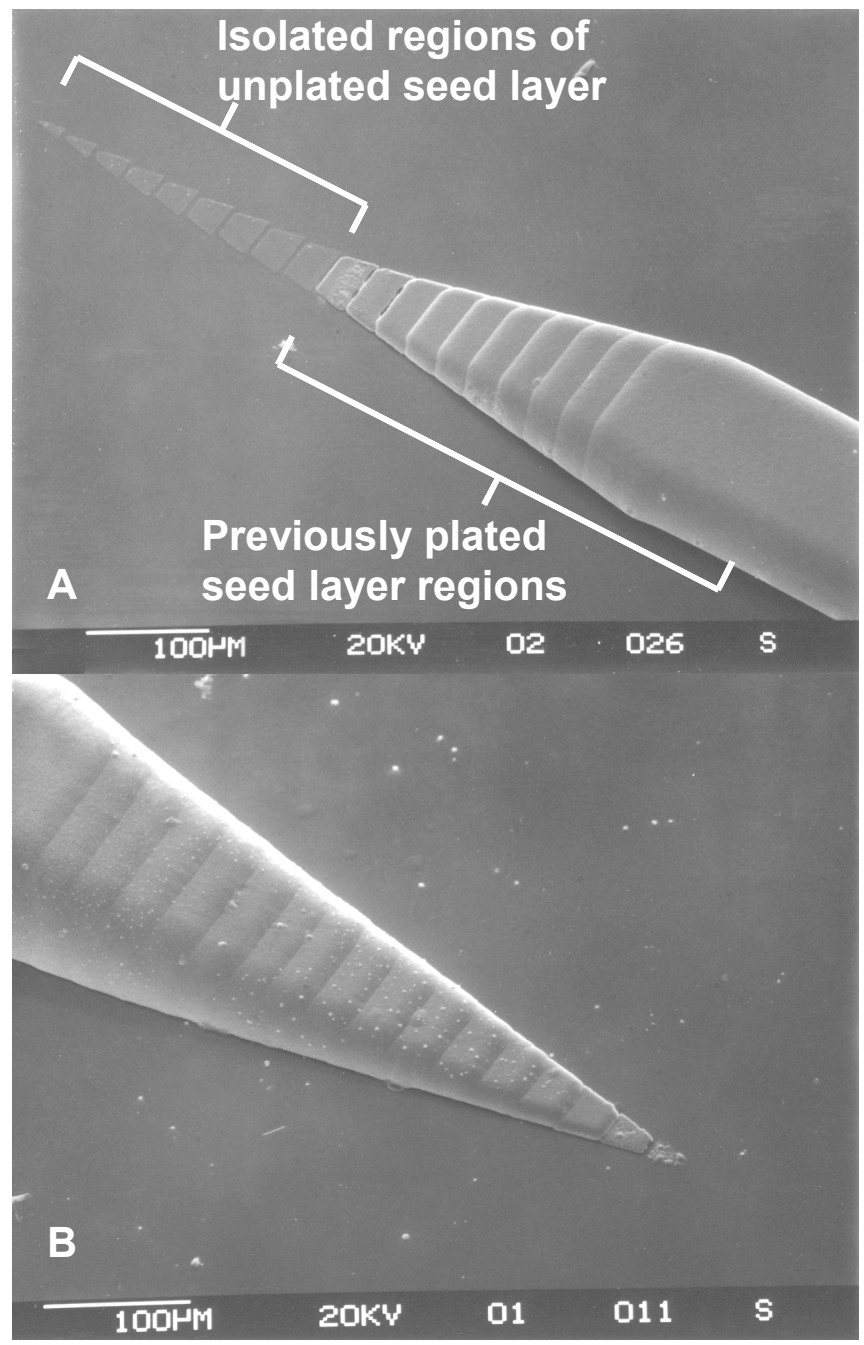

Figure 4. (a) Partially completed 3-D probe plating process.(b) Fully plated tapered probe (a)
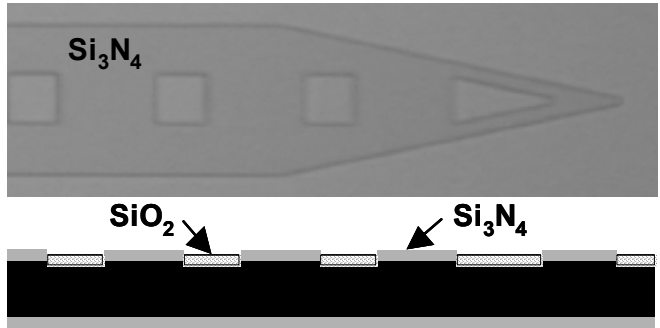

(b)
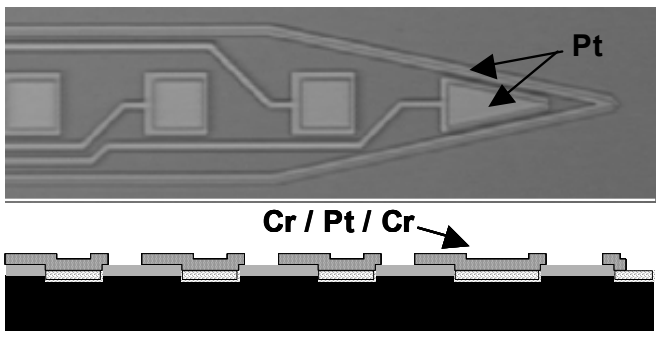

(c)
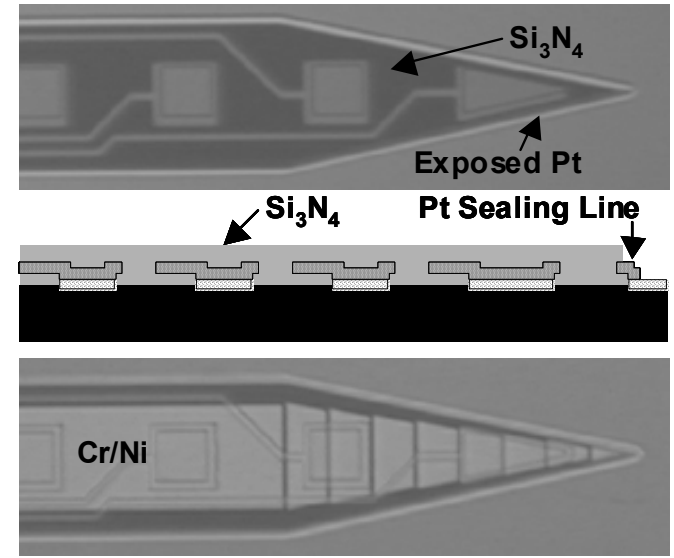

(d)
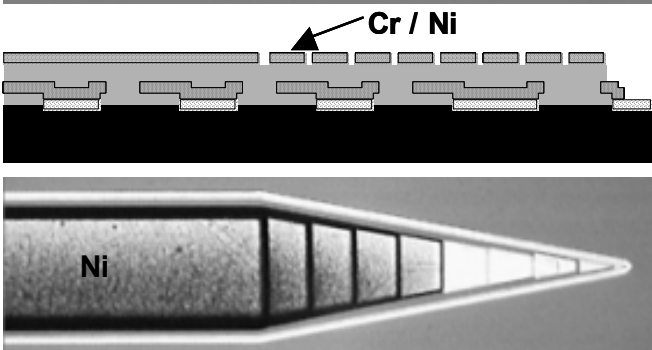

(e)
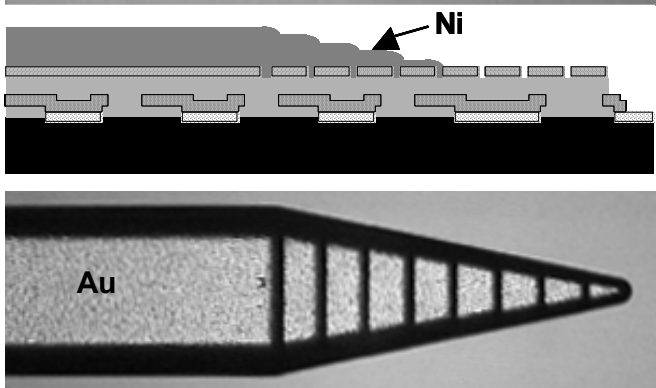

(f)

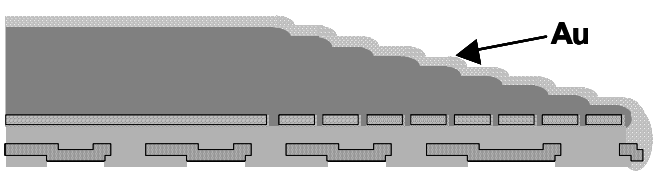

Figure 5. Fabrication process: (a) first insulating layer, (b) interconnect and electrode, (d) second insulating layer, (d) patterned seed layer, (e) electroplated probe shown half way through plating process, (f) plated probe release from substrate. 
The next few steps are used to construct the mechanical supporting layer of the probe. Importantly, the in-plane shape of the probe, as defined by the etched nitride layers, will be retained and the thickness of the mechanical layer will be thin at the tip but gradually becoming thicker and stronger at the other end. To accomplish this 3-D geometry, a patterned seed layer made of $10 \mathrm{~nm}$ of $\mathrm{Cr}$ (adhesion layer) and $100 \mathrm{~nm}$ of $\mathrm{Ni}$ (seed layer) is deposited by electron-beam evaporation on top of the second nitride layer (Figure 5d). As the electrodeposit forms on the region of the patterned seed layer connected to the plating power supply, the film plates upwards and outwards (Figure 4e). When a neighboring island of isolated seed layer is reached, plating commences over this newly connected surface (Figure 4a).

By linking a series of seed layer islands of different shapes and sizes the thickness and width of the resulting shank can be controlled over a wide range. In our process we use electroplated nickel for strength and a subsequent layer of electroplated gold for biocompatibility (Figure 5e).

In the absence of a plating mold, the effective electroplating area for a patterned seed layer deposit increases with time. Since it is desirable to maintain a constant plating current throughout the formation of the mechanical layer, a program was written in Visual Basic to control the power supply (Agilent E3634A) via an RS-232 interface. The current was increased every minute at increments of $\sim 150 \mu \mathrm{A}$. After the plating steps are finished, the probes are released in $\mathrm{KOH}$ at $70^{\circ} \mathrm{C}$ for 15 hours. The overall probe structure consists of platinum electrodes, conductive traces, silicon nitride insulation layers and an electroplated metal shank.

\section{EXPERIMENTAL CHARACTERIZATION}

The microelectrode probes were tested electrically and mechanically. The series resistance of the electrode interconnect is $3.4 \mathrm{k} \Omega$. No shorting was observed between channels and the electroplated mechanical supporting layer. The electrode impedance was tested in artificial cerebral spinal fluid (i.e., a sodium chloride-based saline solution) at room temperature. The impedance value of a typical electrode site is $4.5 \mathrm{k} \Omega$ at a frequency of $10 \mathrm{kHz}$. The electrode was also stimulated with a biphasic charge-balanced $200-\mu$ s-wide pulse at $180 \mathrm{~Hz}$, with an amplitude of $1 \mathrm{~mA}$. At currents higher than $1.5 \mathrm{~mA}$, hydrolysis was observed at the electrode site. Initial mechanical testing showed that when inserted into simulated brain tissue the probes did not bend nor buckle and were able to be inserted accurately.

\section{CONCLUSIONS}

A 22-mm-long multi-channel microprobe electrode array has been designed for DBS application. The design attempts to minimize damage to surrounding tissue while providing sufficient stiffness for this long microprobe to target small brain regions. The DBS microprobe was produced with a low-cost batch-fabrication process that includes a novel 3-D plating process to shape the mechanical supporting layer of the probe into a tapered geometry similar to conventional microwires. Each microfabricated DBS probe has multiple electrodes that can be used to target and tailor the stimulus. These microprobes have the potential to significantly impact DBS research, such as the investigation of treatments for Parkinson's Disease and its debilitating symptoms. In principle, this approach to multielectrode microprobe fabrication could also be combined with integrated circuits, in a batch or assembled process.

\section{ACKNOWLEGMENT}

The authors would like to thank Andy Hung for his assistance with the electrochemical testing and the UCLA Applied MEMS laboratory. This work is supported by the NIH-funded UCLA Morris K. Udall Center for Parkinson's Disease Research under grant NS38367.

\section{REFERENCES}

1. P. D. Wall, W. S. McCulloch, J. Y. Lettvin, and W. H. Pitts, "The terminal arborization of the cat's pyramidal tract determined by a new technique", Yale Journal of Biological Medicine, 28, (1956).

2. F. T. Hambrecht and J. B. Reswick, United States, Rehabilitation Services Administration, and National Institute of Neurological and Communicative Disorders and Stroke, Functional electrical stimulation applications in neural prostheses New York: M. Dekker, (1977).

3. J. G. Nutt, S. L. Rufener, J. H. Carter, V. C. Anderson, R. Pahwa, J. P. Hammerstad, and K. J. Burchiel, "Interactions between deep brain stimulation and levodopa in Parkinson's disease," Neurology, 57, 10 (2001).

4. J. S. Yeomans, Principles of brain stimulation New York: Oxford University Press, (1990).

5. K. Najafi, "Solid-state microsensors for cortical nerve recordings," IEEE Engineering in Medicine and Biology Magazine, 13, 3 (1994).

6. K. Cheung, L. Gun, K. Djupsund, D. Yang, and L. P. Lee, "A new neural probe using SOI wafers with topological interlocking mechanisms," 1st Annual International IEEE-EMBS Special Topic Conference on Microtechnologies in Medicine and Biology (2000).

7. Y. Sugai, F. Asa, Y. Okada, T. Yokoshima, T. Momma, T. Osaka, and T. Ito, "Formation of microprobe using nickel electrodeposition," Electrochemistry, 67, 12 (1999).

8. W. C. Young, R. G. Budynas, and R. J. Roark, Roark's Formulas for stress and strain, 7th ed ed. New York: McGrawHill, (2001).

9. A. Maciossek, SPIE, Semicond.Equipment \& Mater.Int, and NIST, "Electrodeposition of 3D microstructures without moulds," Proceedings of the SPIE - The International Society for Optical Engineering, 2879, (1996). 